\title{
A GENETIC ALGORITHM FOR THE RESOURCE CONSTRAINED PROJECT SCHEDULING PROBLEM (RCPSP)
}

Edgar Gutiérrez Franco, Fernando La Torre Zurita y Gonzalo Mejía Delgadillo

\begin{abstract}
This paper proposes a Genetic Algorithm for the Resource Constrained Project Scheduling Problem (RCPSP). Resources are renewable and there is a unique way to perform the activities. This work employs Genetics Algorithms to schedule project activities to minimize makespan subject to precedence constraints and resources availability. A serial generation scheme is used to obtain the schedule. The algorithm was programmed using Object Oriented programming that allows generating individuals with their own attributes such as activity sequence and makespan. A Genetic Algorithm (GA) is proposed which uses a novel chromosome representation. The issues of the GA parameter tuning are also discussed in this paper. A computer tool that allows the user to define activities, precedence constraints and resource capacity was developed.
\end{abstract}

Keywords: RCPSP, Project Scheduling, Resource Constrained Project, Genetic Algorithms. 